\title{
Nine cases of catamenial pneumothorax: a report of a single- center experience
}

\author{
Chihiro Furuta, Motoki Yano, Hiroki Numanami, Masayuki Yamaji, Rumiko Taguchi, Masayuki Haniuda \\ Division of Chest Surgery, Department of Surgery, Aichi Medical University, Nagakute, Japan \\ Contributions: (I) Conception and design: M Yano; (II) Administrative support: M Haniuda; (III) Provision of study materials or patients: M Yamaji, \\ R Taguchi; (IV) Collection and assembly of data: C Furuta, H Numanami; (V) Data analysis and interpretation: C Furuta, H Numanami; (VI) \\ Manuscript writing: All authors; (VII) Final approval of manuscript: All authors. \\ Correspondence to: Motoki Yano, MD, PhD. Division of Chest Surgery, Department of Surgery, Aichi Medical University, 1-1 Yazakokarimata, \\ Nagakute 480-1195, Japan. Email: motoki2ds@yahoo.co.jp.
}

Background: Catamenial pneumothorax (CP) is defined as repeated pneumothorax related to menses and thoracic endometriosis. We performed a retrospective analysis of nine patients with CP to determine the clinical features as well as the effects of treatment and recurrence rates.

Methods: A retrospective review was conducted of the clinical and pathologic data in all CP patients undergoing treatment at our institution. Nine patients underwent treatment for CP. Of these, six underwent surgical treatment 8 times.

Results: The median age was 36 years. Six patients had experienced delivery. The laterality of the pneumothorax was right in all patients. Pelvic endometriosis was diagnosed in five patients. Six patients underwent surgical treatment. Partial resection of the lung was performed in four patients and partial resection of the diaphragm in five. Of these, both resections were performed in four patients. A pathological diagnosis of endometriosis was achieved in only three patients. The observation period was 16.7 months. In the six patients with surgical resection, five experienced recurrence at various intervals. Onset of pneumothorax occurred pre- or menstrual period in most cases.

Conclusions: The diagnosis and treatment of CP is not easy. A multidisciplinary approach and skillful management are required. Recurrence of $\mathrm{CP}$ is common following a temporary cure of pneumothorax by surgical treatment.

Keywords: Catamenial pneumothorax $(\mathrm{CP})$; thoracic endometriosis; menses

Submitted Mar 22, 2018. Accepted for publication Jul 01, 2018.

doi: $10.21037 /$ jtd.2018.07.39

View this article at: http://dx.doi.org/10.21037/jtd.2018.07.39

\section{Introduction}

Catamenial pneumothorax (CP) is defined as spontaneous recurrent pneumothorax and occurs in women of reproductive age with a temporal relationship with menses. It was first reported in 1958 by Maurer et al. (1). This unique entity of spontaneous recurring pneumothorax in reproductive women has been investigated for the past seven decades (1-13). Recent attempts have been made to classify thoracic endometriosis-related pneumothorax as catamenial and no-catamenial thoracic endometriosis- related pneumothorax $(2,3)$. Despite improvements in the recognition of this rare entity, the diagnosis of $\mathrm{CP}$ is not easy, and its optimum management remains unclear. We performed a retrospective analysis of nine patients with $\mathrm{CP}$ to determine the clinical features as well as the effect of treatment and recurrence rates.

\section{Methods}

A retrospective review of the clinical and pathologic data in all $\mathrm{CP}$ patients undergoing treatment at Aichi Medical 


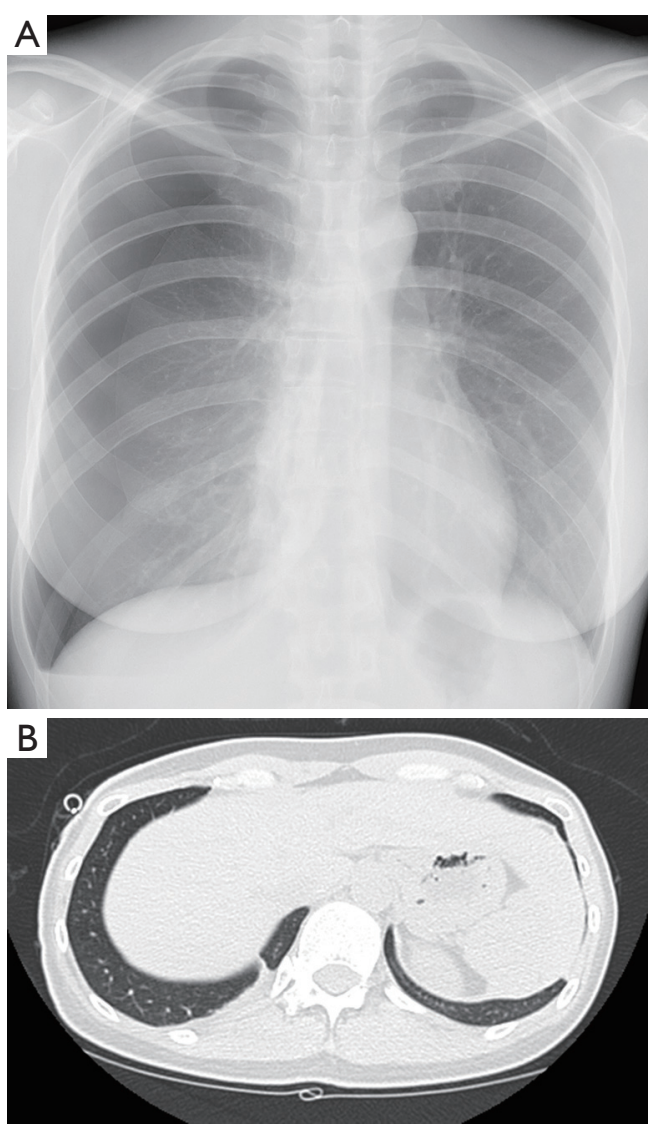

Figure 1 Preoperative radiological findings in case 9. (A) Chest $\mathrm{X}$-ray photography; (B) chest CT at the level of the diaphragm.

University Hospital between March 2003 and November 2016 was conducted. The study was approved by the Institutional Review Board of Aichi Medical University Hospital (No. 2016-H134). During this time period, nine patients underwent treatment for CP. Of these, six patients underwent surgical treatment eight times.

\section{Diagnosis of CP}

$\mathrm{CP}$ was diagnosed as spontaneous recurrent pneumothorax (Figure 1: chest X-ray photography: case 9) in women of reproductive age with a temporal relationship with menses. In the patients with a first diagnosis of pneumothorax, $\mathrm{CP}$ was diagnosed based on recurrent chest pain and/or discomfort. The diagnosis of CP was divided into clinical, surgical and pathological diagnoses. The patients who were diagnosed with only a clinical record without surgical resection of the lung or diaphragm were defined as clinically diagnosed CP. A pathological diagnosis of $\mathrm{CP}$ was given to the patients with endometriosis pathologically diagnosed in resected specimens. If no endometriosis was found in the partially resected lung or diaphragm with findings suspicious of $\mathrm{CP}$, a surgical diagnosis was given.

\section{Results}

The clinical and pathological data of all none patients are listed in Table 1. The median age was 36 years (range, 26-42 years). Six of the 9 patients had experienced delivery (1 to 3 times). The laterality of the pneumothorax was right in all patients. Pelvic endometriosis was diagnosed in five patients. Three patients underwent hormonal treatment [gonadotrophin-releasing hormone (GnRH)] analogue or dienogest, a steroidal progestin of progesterone receptor agonist) before the consultation at our department. In three patients, hormonal treatment was selected as the principal treatment without surgical treatment, and pneumothorax was treated only with chest drainage or observation.

Six patients underwent surgical treatment. Partial resection of the lung was performed in four patients and partial resection of the diaphragm in five. Of these, both resections were performed in four patients. In all five patients who underwent diaphragm resection, circular red spots and/or small holes were recognized on the central tendon of the diaphragm (Figure 2A). In 3 of the 4 patients who underwent pulmonary resection, red or black spots of endometriosis were recognized on the visceral pleura of the lower lobe (Figure 2B). In two patients with pulmonary resection, the bulla in S1 (case 3) and S5 (case 5) was resected. A pathological diagnosis of endometriosis was obtained on the specimens of the diaphragm in only three patients (No. 4, 6, and 9) (Figure 3). The observation period was 16.7 months. Of the 6 patients with surgical resection, 5 experienced recurrence with various intervals (1 to 19 months). Of these five patients, additional surgical treatment was performed in 2. Both patients underwent pulmonary resection because air leakage was strongly suspected from the pulmonary tissue. Diaphragmatic coverage by oxidized regenerated cellulose (ORC) mesh was added in a patient because microscopic fistula was suspected on the surface of the diaphragm.

As three patients were followed up without surgical treatment, conservative treatment was selected. Pleural drainage was performed in one patient. But 2 of 3 patients refused even pleural drainage. Several times of recurrence occurred in all three patients even with hormonal therapy.

We have assessed the relation between the onset of 


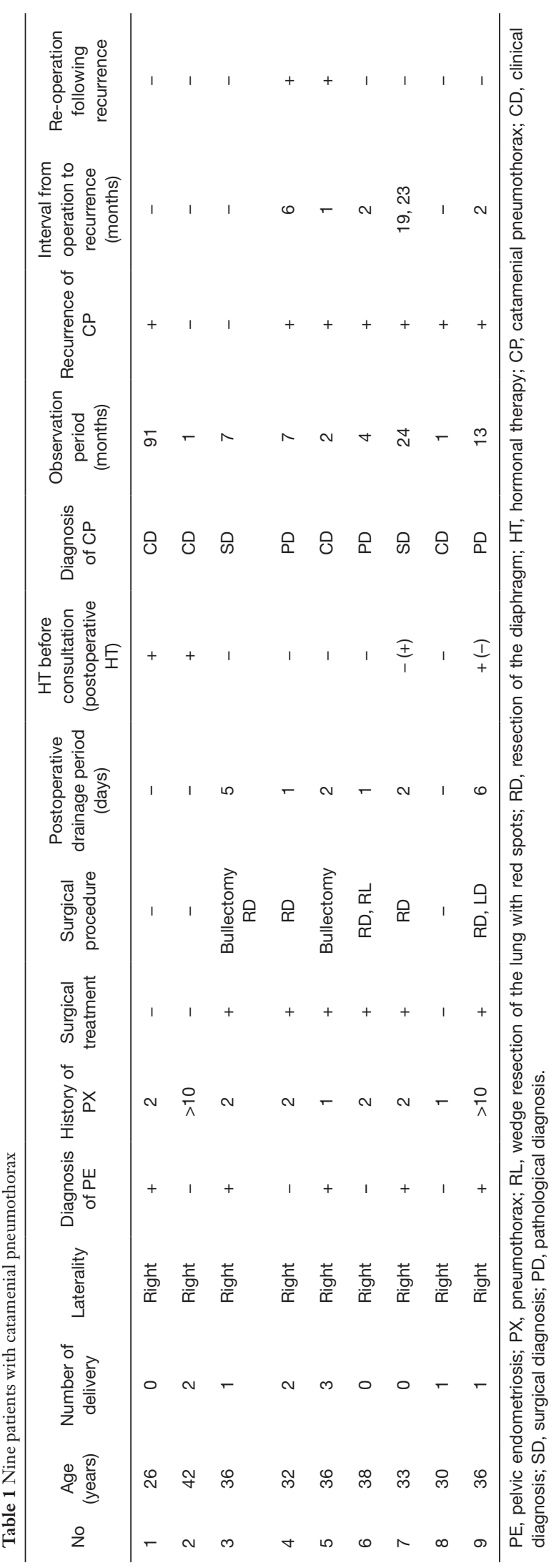

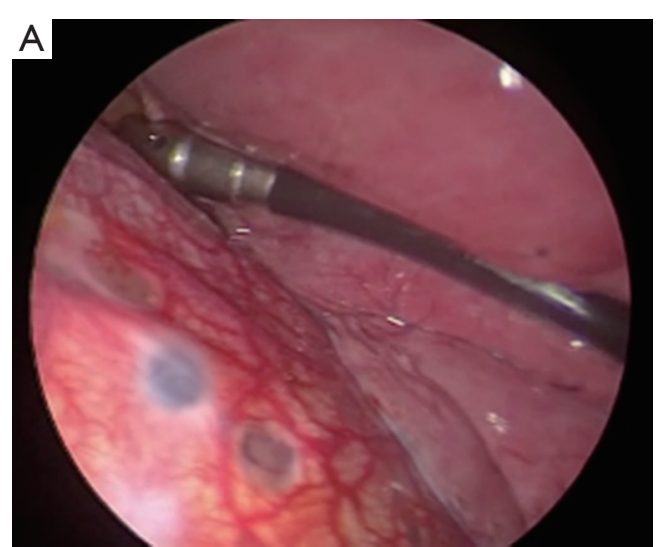

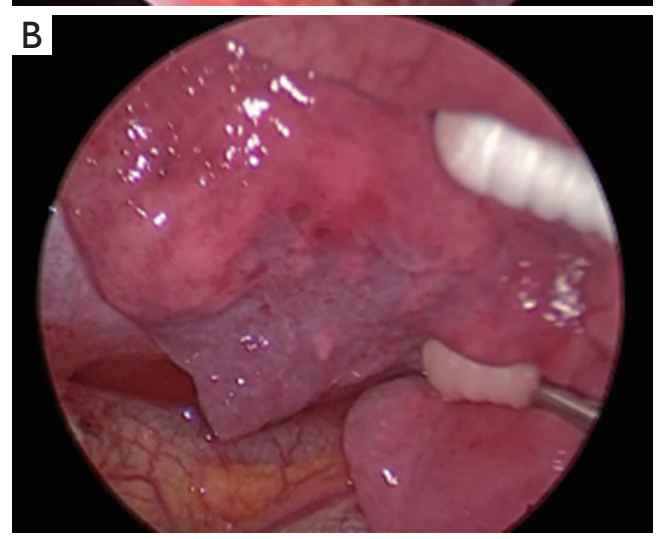

Figure 2 Intraoperative findings in case 9. (A) Circular red spots on the central tendon of the diaphragm; (B) circular red spots on the visceral pleura of the lower lobe.

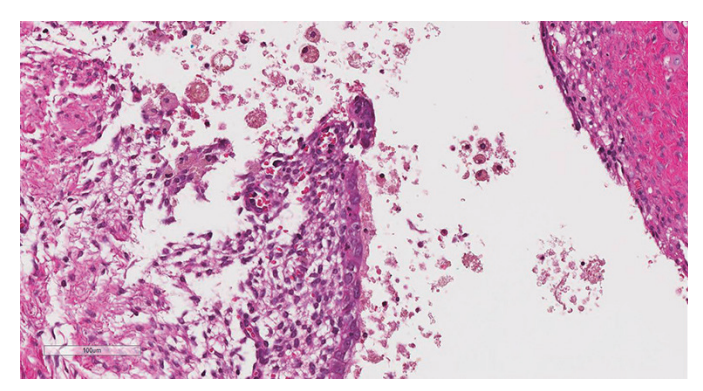

Figure 3 Pathological findings of catamenial pneumothorax (case 9). The findings of endometriosis were obvious on the diaphragm, which showed uterus stroma, hemorrhaging, and macrophages (HE staining, $\times 100$ magnification). The white bar scales $100 \mu \mathrm{m}$.

pneumothorax and the menstruation cycle in Figure 4. Only the date which clearly recorded was tabulated. They were scattered without obvious peak of onset. However, the onset of pneumothorax occurred pre- or menstrual period in most cases (13/15, including recurrences). 


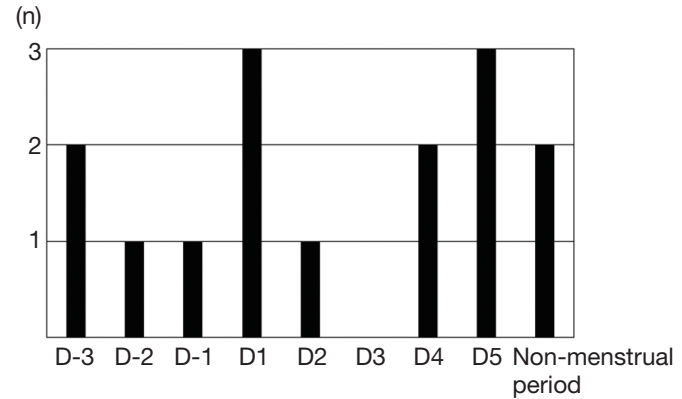

Figure 4 Distribution of the onset of pneumothorax and the menstrual cycle. D1: day 1 of the cycle, D-1: 1 day before D1.

\section{Discussion}

In this study, we investigated the clinical features of $\mathrm{CP}$ and recognized a high recurrence rate following surgical treatment. Our findings do not support the usefulness of surgical treatment for preventing recurrence of CP. Surgical treatment for CP was mainly selected to cure the air leakage of pneumothorax that had not been cured by chest drainage. Although the diaphragm was covered with sheets of polyglycolic acid (4) and pleurodesis $(5,6)$ to reduce the risk of recurrence, $\mathrm{CP}$ recurred at a high rate. We believe that the primary purpose of surgical treatment is to cure the air leakage of pneumothorax that was not cured by chest drainage.

The postoperative recurrence rates have varied in former reports. Subotic et al. (7) reported no recurrent cases. Attaran et al. reported only recurrent case in 12 operated cases (14). Meanwhile, Haga et al. reported that almost half of patients (49\%) recurred (15). The differences of surgical treatment chosen by the authors may influenced to the various recurrence rates. Wide diaphragmatic resection and grafting have been reported to have a low recurrence rate. Haga reported higher diagnosis of endometrial gland in the diaphragm in the recurrent group. If so, postoperative hormone therapy must be important to prevent recurrence. In a series of low recurrence rate, Attaran et al. reported all patients received hormone therapy postoperatively. However, it is not easy for young females to accept hormone therapy for a long time. CP recurred following the interruption of hormonal treatment for the purpose of becoming pregnant in a case. In our study, 5 of the 6 patients who underwent surgery did not undergo postoperative hormonal therapy for various reasons, even though hormonal treatment seems to be the most effective treatment for reducing the risk of recurrence, as reported by many authors $(5,8)$.

Thoracic observation and tissue sampling is another reason for performing surgery. Video-assisted thoracic surgery (VATS) has been often performed recently (5). This technique has enabled detailed thoracic observations. Surgical treatment can be useful both to cure pneumothorax and to obtain pathological specimens. However, it is difficult to obtain pathological findings of endometriosis in this manner. Indeed, in our study, characteristic pathological findings were noted in only two patients. We observed no characteristic findings even with meticulous inspection of the thorax. The presence of such findings seems to depend on the stage of the disease and the size and number of characteristic lesions. In addition, the timing of the operation seems to be important. Bagan et al. suggested the effectiveness of surgical treatment during menses, as endometriotic lesions were visualized more easily during this time (9). Alifano et al. reported the usefulness of repeated surgery for recurrent pneumothorax after surgery in women, frequently observing endometriotic findings that had been initially missed (10). Slasky et al. reported the usefulness of pneumoperitoneum for identifying otherwise invisible diaphragmatic holes (11). We have never performed such procedures, though, as they seem to induce excessive stress on patients.

In the present study, CP occurred on the right side in all patients. The laterality of $\mathrm{CP}$ has been investigated, and $\mathrm{CP}$ is considered to occur on the right side most frequently. Inoue et al. interestingly reported the relationship between the laterality of $\mathrm{CP}$ and age (12). As the ratio of elderly patients has increased, the predominance of right-sided has become remarkable. In the present study, 8 of 9 patients were in their $30 \mathrm{~s}$ or $40 \mathrm{~s}$, so a high predominance of the right side was expected.

Rousset-Jablonski et al. reported the beautiful peak of onset of pneumothorax on the first day of the menstruation cycle (16). We have also assessed the distribution of the onset of pneumothorax according to the menstruation cycle. Unfortunately, we could not find such clear relation between the onset of pneumothorax and the menstruation cycle. However, it is difficult to evaluate the onset of pneumothorax accurately because the patients' memory of symptoms was not clear.

\section{Conclusions}

The diagnosis and treatment of CP is not easy. A multidisciplinary approach and skillful management are 
required. The primary purpose of surgical treatment to cure the air leakage of pneumothorax has been achieved. However, recurrence of CP is common even following a temporary cure of pneumothorax by surgical treatment. Adjuvant hormonal treatment is strongly recommended. As such, the maintenance of hormonal therapy will be the next issue to tackle for women hoping to become pregnant.

\section{Acknowledgements}

None.

\section{Footnote}

Conflicts of Interest: The authors have no conflicts of interest to declare.

Ethical Statement: The study was approved by the Institutional Review Board of Aichi Medical University Hospital (No. 2016-H134).

\section{References}

1. Maurer ER, Schaal JA, Mendez FL Jr. Chronic recurring spontaneous pneumothorax due to endometriosis of the diaphragm. J Am Med Assoc 1958;168:2013-4.

2. Fukuoka M, Kurihara M, Haga T, et al. Clinical characteristics of catamenial and non-catamenial thoracic endometriosis-related pneumothorax. Respirology 2015;20:1272-6.

3. Alifano M, Jablonski C, Kadiri H, et al. Catamenial and noncatamenial, endometriosis-related or nonendometriosis-related pneumothorax referred for surgery. Am J Respir Crit Care Med 2007;176:1048-53.

4. Takahashi M, Matsukura T, Hirai T, et al. Recurrent catamenial hemopneumothorax treated by coverage with polyglycolic acid sheets. J Thorac Cardiovasc Surg 2013;145:300-2.

5. Korom S, Canyurt H, Missbach A, et al. Catamenial

Cite this article as: Furuta C, Yano M, Numanami H, Yamaji M, Taguchi R, Haniuda M. Nine cases of catamenial pneumothorax: a report of a single-center experience. J Thorac Dis 2018;10(8):4801-4805. doi: 10.21037/jtd.2018.07.39 pneumothorax revisited: clinical approach and systematic review of the literature. J Thorac Cardiovasc Surg 2004;128:502-8.

6. Ciriaco P, Negri G, Libretti L, et al. Surgical treatment of catamenial pneumothorax: a single centre experience. Interact Cardiovasc Thorac Surg 2009;8:349-52.

7. Subotic D, Mikovic Z, Atanasijadis N, et al. Hormonal therapy after the operation for catamenial pneumothorax is it always necessary? J Cardiothorac Surg 2016;11:66.

8. Hwang SM, Lee CW, Lee BS, et al. Clinical features of thoracic endometriosis: A single center analysis. Obstet Gynecol Sci 2015;58:223-31.

9. Bagan P, Le Pimpec Barthes F, Assouad J, et al. Catamenial pneumothorax: retrospective study of surgical treatment. Ann Thorac Surg 2003;75:378-81; discusssion 381.

10. Alifano M, Legras A, Rousset-Jablonski C, et al. Pneumothorax recurrence after surgery in women: clinicopathologic characteristics and management. Ann Thorac Surg 2011;92:322-6.

11. Slasky BS, Siewers RD, Lecky JW, et al. Catamenial pneumothorax: the roles of diaphragmatic defects and endometriosis. AJR Am J Roentgenol 1982;138:639-43.

12. Inoue $T$, Chida $M$, Inaba $H$, et al. Juvenile catamenial pneumothorax: institutional report and review. J Cardiothorac Surg 2015;10:83.

13. Visouli AN, Zarogoulidis K, Kougioumtzi I, et al. Catamenial pneumothorax. J Thorac Dis 2014;6:S448-60.

14 Attaran S, Bille A, Karenovics W, et al. Videothoracoscopic repair of diaphragm and pleurectomy/abrasion in patients with catamenial pneumothorax: a 9-year experience. Chest 2013;143:1066-9.

15 Haga T, Kurihara M, Kataoka H, et al. Clinicalpathological findings of catamenial pneumothorax: comparison between recurrent cases and non-recurrent cases. Ann Thorac Cardiovasc Surg 2014;20:202-6.

16. Rousset-Jablonski C, Alifano M, Plu-Bureau G, et al. Catamenial pneumothorax and endometriosis-related pneumothorax: clinical features and risk factors. Hum Reprod 2011;26:2322-9. 